\title{
ATIVIDADE BASEADA EM PROBLEMA APLICADA NA DISCIPLINA DE SISTEMAS HIDRÁULICOS E PNEUMÁTICOS: METODOLOGIA SATC 2030
}

Yan Iuri Fagundes Cardoso - y.fagundes@ hotmail.com

Marina Souza Costa - marinasouzacosta@ hotmail.com

Augusto Mandelli - mandelli.augusto@gmail.com

Luiz Eduardo dos Santos Bif - luizeduardo.bif@gmail.com

Richard de Medeiros Castro - richard.castro@satc.edu.br

Faculdade SATC, Engenharia Mecânica. Rua Pascoal Meller, 73 - Universitário, 88805-380,

Criciúma, SC.

Resumo: A metodologia de aprendizagem da Faculdade SATC propõe aos professores que esta prática deve ser concebida em um ambiente colaborativo e de cocriação, com intuito de melhores práticas para a inovação no ensino superior. Esta proposta está relacionada com a nova conjuntura global das tecnologias educacionais, da quebra de paradigmas da educação tradicional e, das novas competências e habilidades requeridas pelo mercado de trabalho. Com esse propósito, a cada semestre, grupos de acadêmicos em sua respectiva disciplina, são encorajados na resolução de problemas reais, auxiliados constantemente pelo professor, que compartilha de suas experiências profissionais na resolução desses problemas. Nesse trabalho, os acadêmicos tiveram que avaliar o uso de um acumulador de pressão em um sistema hidráulico que opera em condições de fluxo intermitente e, de alto consumo de energia. O trabalho foi desenvolvido na disciplina de sistemas hidráulicos $e$ pneumáticos, ministrada no oitavo semestre do curso de engenharia mecânica. Ao final, os acadêmicos responderam um questionário, para validar quali-quantitativamente a aplicação da metodologia, avaliando o impacto na aprendizagem. No aspecto geral, a atividade de ensino proporcionou a resolução de um problema real, aplicando o conhecimento da área específica, na melhoria da eficiência energética do sistema. Também, a metodologia de aprendizagem baseada em problema, demonstram-se como uma evolução positiva aos métodos tradicionais, e que promove o desenvolvimento da autonomia dos discentes.

Palavras-chave: Metodologia de aprendizagem. Inovação no ensino. Sistemas hidráulicos. Acumulador de pressão. Eficiência energética.

\section{INTRODUÇÃO}

De forma geral, ocorre por meio das instituições e dos docentes, uma constante busca pela evolução das metodologias de ensino, sempre com intuito de melhorar a compreensão das questões abordadas, promovendo maiores habilidades e competências adquiridas pelos acadêmicos. Sendo assim, uma das práticas desenvolvidas por algumas instituições de ensino, trata-se da chamada "atividade baseada em problema" que, de forma resumida, propõe um desafio aos acadêmicos, onde eles devem, por meio de suas técnicas até então adquiridas, gerar uma solução para tal. 
A aprendizagem baseada em problema, do inglês Problem Based Learning $(P B L)$, é definida por Sadlo (2014), como o processo de aprender durante a tentativa de solução ou administrar um problema. Parson e Bignell (2011) explica que PBL toma lugar no que diz respeito a tarefas voltadas aos cursos ou ao mundo real, relacionando o conteúdo da problemática ao material de temática do curso. De acordo com Titisari, Wijayanti e Chomsatun (2013), as conquistas de conhecimentos pelos discentes de forma mais autônoma se sobressai aos métodos convencionais de ensino.

Conforme Souza e Dourado (2015), atualmente, um dos maiores estímulos da educação se situa em levantar transformações no aprendizado, de forma que acompanhem o ritmo de desenvolvimento dos aspectos econômicos, sociais, culturais, ambientais e tecnológicos, contribuindo para a elaboração de uma sociedade com maior equilíbrio social e econômico.

Conhecendo a singularidade de todos e quaisquer indivíduos, observa-se a heterogeneidade de perfis sociais dos alunos, de forma que, o modelo tradicional de ensino não possibilita que tais indivíduos desenvolvam suas proficiências e trabalhem suas dificuldades. Assim, abordar e dividir as tarefas de forma que os discentes possam trabalhar suas competências e superar suas dificuldades, auxilia na obtenção melhoria do aprendizado individual, em grupo e autônomo.

Alinhando conceitos de aprendizagem baseada em problema com as estratégias empreendedoras, inovação social, necessidades da comunidade, oportunidades, tendências e alguns dos objetivos do desenvolvimento sustentável, fora elaborada a metodologia SATC 2030, que abrange todos os acadêmicos de ensino superior da instituição, trabalhando soft skills e as questões multifatoriais do aprendizado (uso de tecnologia, trabalho em conjunto, tomada de decisão, aplicação prática, etc.).

O presente trabalho tem como propósito avaliar a junção de aprendizagem baseada em problemas aplicada a disciplina de sistemas hidráulicos e pneumáticos, usando um problema real de contexto energético, para desenvolver um conteúdo específico, tradicionalmente visto de forma expositiva. Neste aspecto, foi proposto o uso de acumuladores de pressão como fonte de energia auxiliar para a resolução do problema, e os resultados e impactos tecnológicos e da metodologia de ensino serão avaliados. A priori, abordaram-se as condições desempenho do conjunto com e sem o emprego de um acumulador, com enfoque no consumo de potência necessário pela bomba, e por fim, serão colhidos os feedbacks dos alunos participantes da disciplina com respeito as habilidades desenvolvidas e qual a relevância da metodologia para o aprendizado nas competências da disciplina.

\section{ATIVIDADE DE ENSINO E PROBLEMA PROPOSTO}

Como atividade, foi proposto o desenvolvimento do conhecimento específico, elaborando e recordando modelos matemáticos, desenvolvido em outras disciplinas do curso de engenharia mecânica. É esperado que o discente trabalhe as habilidades de emprego de softwares, justificando suas escolhas e resultados de cálculos.

A atividade proposta surgiu a partir da identificação de um problema em um sistema hidráulico, onde o mesmo, quando em operação, deveria trabalhar com ciclo conforme a Figura 1b. Nesse caso, realizou-se uma análise do ciclo e propuseram-se algumas alternativas para a redução do consumo de energia, principalmente nos instantes de maiores picos que ocorrem nos períodos 10 a 20 s e de 35 a 45 s, sabendo que, o sistema hidráulico deveria operar com pressão mínima de 160 bar. Na Figura 1 os principais componentes do circuito hidráulico e o ciclo de operação da máquina em questão são identificados. 
Figura 1 - Sistema hidráulico proposto: a) componentes do circuito hidráulico e

b) ciclo de operação do sistema hidráulico
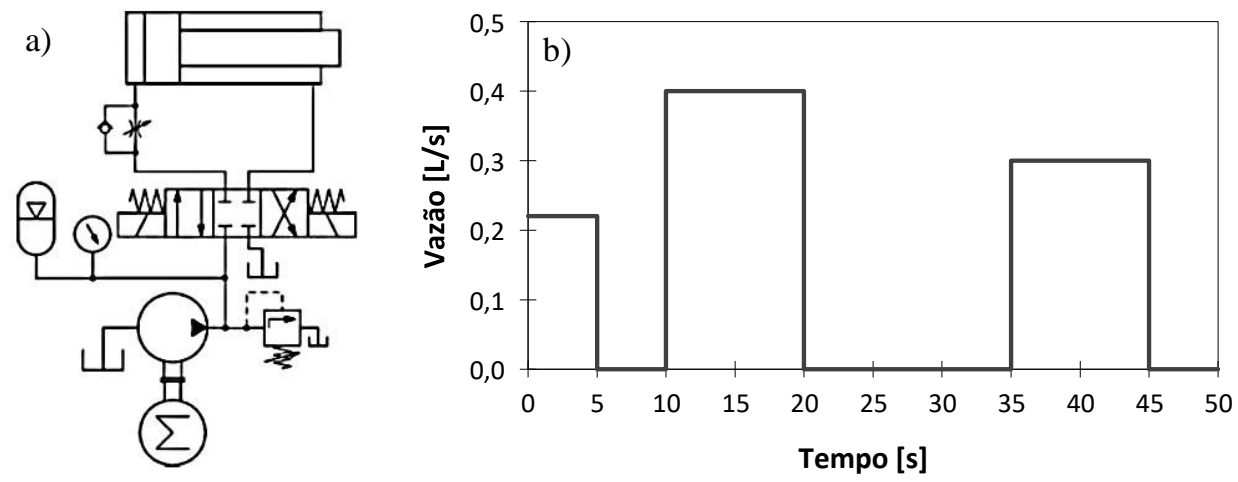

Fonte: dos autores (2020)

Por meio da Figura 1b é possível identificar características de um ciclo intermitente, no qual altas vazões são requisitadas em curto período, totalizando um ciclo de 50 segundos. Em função de tais atributos do sistema, é notável que determinados componentes (como: bomba e motor) tornam-se superdimensionados quando em situações menos demandantes do ciclo, em detrimento do funcionamento nos instantes com maior vazão.

Em busca do aperfeiçoamento de desempenho do sistema, uma melhor eficiência energética é indispensável, dessa forma, como sugerido por Castellanos (2017) e Linsingen (2001), se torna possível a aplicação de acumuladores hidropneumáticos, onde a bomba supre fluido para tal componente em períodos de baixa demanda e posteriormente, ambos elementos suprem os altos fluxos demandados pelo ciclo. Sendo assim, para determinar o impacto da aplicação do acumulador hidropneumático, se faz necessária a análise das condições de operação do sistema.

\section{RESOLUÇÃO DO PROBLEMA}

Para o correto dimensionamento do acumulador de pressão, foi necessário também redefinir alguns componentes do circuito hidráulico. A fim de comparação de consumo de energia do circuito hidráulico, ou seja, com e sem o uso do acumulador, cálculos foram realizados para a seleção da bomba hidráulica, em seguida o acumulador de pressão.

\subsection{Dimensionamento da bomba sem uso de acumulador}

Os dados de entrada apresentados na Tabela 1, são provenientes do problema proposto, onde a $P_{\text {máx }}$ representa a maior pressão e $Q_{\text {máx }}$ a maior vazão presente no sistema.

Tabela 1 - Dados de entrada para o cálculo da potência de acionamento

Fonte: dos autores (2020).

\begin{tabular}{c|c|}
\hline$Q_{\text {máx }}[\mathrm{L} / \mathrm{min}]$ & $P_{\text {máx }}[\mathrm{bar}]$ \\
\hline 24 & 240 \\
\hline
\end{tabular}

Seguindo a rotina proposta pelo catálogo de bombas Parker (2012), primeiramente se faz necessário calcular o deslocamento volumétrico da bomba $\left(V_{g}\right)$ por meio da Equação (1). Dessa forma, foi calculada então a vazão teórica da bomba por meio da Equação (2): 
C.COBENCE 2020

"Os desafios para formar hoje o engenheiro do amanhã"

$$
\begin{gathered}
V_{g}=\frac{1000 Q_{\operatorname{máx}}}{n} \\
Q_{\text {teórico }}=\frac{V_{g} n}{1000}
\end{gathered}
$$

A quantidade de vazamento interno (internal leakage) é representada na Figura 2 a), encontrada no catálogo Parker (2005) de bomba de palhetas. Também apresentada por Parker (2005), a Figura 2 b) proporciona a aquisição de perda de potência para a bomba.

Logo, foi possível determinar a potência teórica da bomba $N_{\text {teórica }}$ por meio das Equações (3) e (4). Em sequência, a Equação (5) entrega a potência real $N_{\text {real }}$ para acionamento da bomba, chegando à potência de 10,2 kW, encontrando o modelo T7AS B17.

Figura 2 - Curvas de desempenho da bomba: a) vazamento interno da bomba e b) perda de potência da bomba
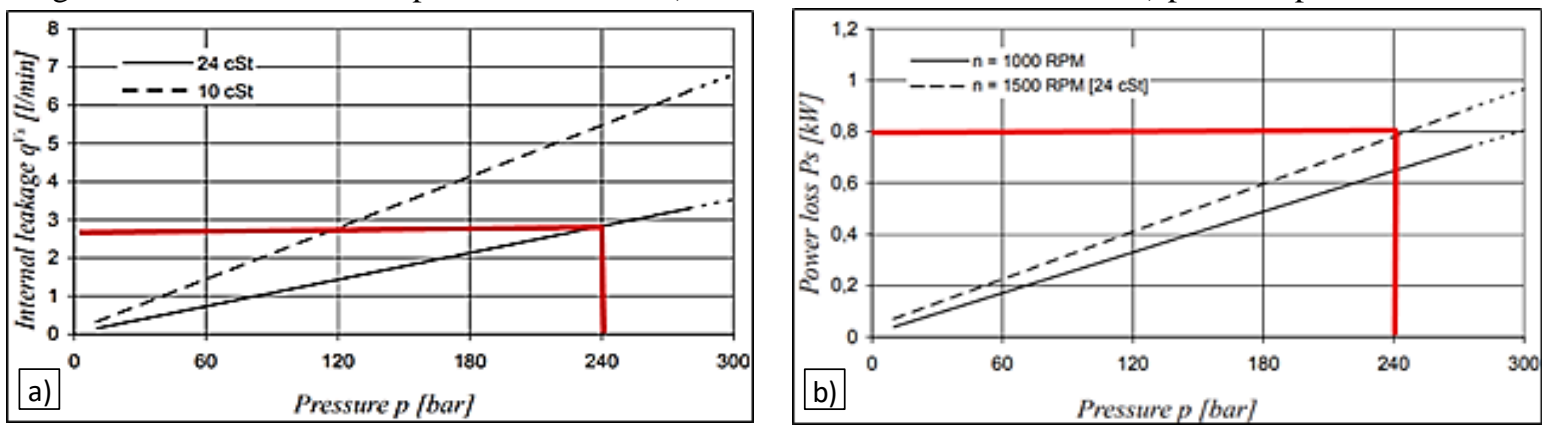

Fonte: Parker (2005)

$$
\begin{aligned}
& Q_{e f f}=Q_{\text {teórico }}-q_{v s} \\
& P_{\text {teórico }}=\frac{Q_{\text {teórico }} p}{600} \\
& P_{\text {real }}=P_{\text {teórico }}+P_{s}
\end{aligned}
$$

\subsection{Definição do tamanho teórico para o acumulador de pressão}

Efetuou-se o cálculo de vazão média que a bomba precisa suprir para o circuito, seguido pela verificação de variação de volume de fluido no acumulador, chegando a um volume mínimo necessário para suprir as demandas do circuito. A vazão média $\left(Q_{m e d}\right)$ suprida pela bomba é dada pela Equação (6), proposta por Linsingen (2016).

$$
Q_{m e d}=\frac{\sum Q \cdot t}{\sum t}
$$

Considerando um ciclo de 50 segundos, a vazão média resulta em 9,72 L/min. A Tabela 2 proporciona a avaliação da variação de volume em todos os instantes do ciclo.

A Tabela 2 também percebe-se que a maior variação de volume (Vx), ocorre entre $20 \mathrm{e}$ 35 segundos, referente a 2,43 litros. Tal valor associa-se a variação de fluido no acumulador na pressão mínima de trabalho e o volume na pressão máxima, demonstrado pela Equação (7), apresentada por Linsingen (2016). 
Tabela 2 - Variação de volume em intervalos do ciclo

\begin{tabular}{c|c|c|}
\hline Item & Intervalos $[\mathrm{s}]$ & $\Sigma V_{0}$ \\
\hline 1 & 0 & 0 \\
\hline 2 & $0-5$ & $-0,29$ \\
\hline 3 & $5-10$ & 0,52 \\
\hline 4 & $10-20$ & $-1,86$ \\
\hline 5 & $20-35$ & 0,57 \\
\hline 6 & $35-45$ & $-0,81$ \\
\hline 7 & $45-50$ & 0 \\
\hline
\end{tabular}

Fonte: dos autores (2020).

$$
V_{x}=V_{3}-V_{2}
$$

Um acumulador que opera com gás inerte, como o nitrogênio, pode ser avaliado a partir da Lei de Boyle, dada pela Equação (8), apresentada por Linsingen (2016), onde o expoente politrópico $(n)$ deve ser considerado 1,4, de acordo com Fialho (2004).

$$
P_{1} \cdot V_{1}^{n}=P_{2} \cdot V_{2}^{n}=P_{3} \cdot V_{3}^{n}
$$

O dimensionamento do volume teórico do acumulador pode utilizar da Equação (9), dada por Linsingen (2016) para acumuladores como fonte de potência auxiliar/emergência.

$$
V_{1}=\frac{V_{x} \cdot\left(\frac{P_{3}}{P_{1}}\right)^{1 / n}}{1-\left(\frac{P_{2}}{P_{4}}\right)^{1 / n}}
$$

De acordo com o catálogo da fabricante HYDAC a pressão de pré-carga, $P_{1}$ deve ser menor que a pressão mínima de operação, $P_{3}$. Sendo assim, definiu-se que $P_{1}$ seria $90 \%$ de $P_{3}$. As pressões usadas foram, $\mathrm{P} 1=144$ bar, $\mathrm{P} 2=240$ bar, $\mathrm{P} 3=160$ bar o volume de pré carga, V1 = 10,42 L. Por indicação da fabricante (HYDAC), faz-se necessárias duas correções no volume devido às variações de temperatura e de pressão. Estas considerações são apresentadas pelas Equações (10) e (11):

$$
\begin{gathered}
V_{1, T}=V_{1} \cdot \frac{T_{2}}{T_{3}} \\
V_{1, R}=V_{1, T} \cdot C_{a}
\end{gathered}
$$

A Equação (10) considera a máxima e a mínima temperatura de trabalho. Já a Equação (11) recomenda a utilização de um fator de correção adiabática, $C_{a}$, obtido a partir do catálogo do fabricante. Os valores de volume obtidos são expostos na Tabela 3 para efeito de comparação, sendo que, $V_{l, R}$ é o valor real considerado na escolha do acumulador.

Tabela 3 - Volumes definidos para cada condição

\begin{tabular}{c|c|c|}
\hline$V_{1}[\mathrm{~L}]$ & $V_{1, T}[\mathrm{~L}]$ & $V_{1, R}[\mathrm{~L}]$ \\
\hline 10,42 & 14,25 & 17,39 \\
\hline
\end{tabular}

Fonte: dos autores (2020). 


\subsection{Definição da bomba com uso do acumulador de pressão}

A bomba agora foi então selecionada pela vazão média, o que proporciona a diminuição da potência de acionamento. Considerando essa vazão calculada pela Equação (6), bem como a rotação adotada anteriormente, o volume geométrico necessário é dado pela Equação (12)

$$
V_{g}=\frac{1000 \cdot Q_{m e d}}{n}
$$

Seguindo novamente o que propõe Parker (2012), adota-se um valor para o deslocamento geométrico ligeiramente acima do calculado pela Equação (12). Por meio da Tabela 4 e da Equação (5), é encontrada uma potência de acionamento da bomba de 6,68 $\mathrm{kW}$, e o modelo comercial T7AS B10.

Tabela 4 - Dados de entrada do acionamento para cálculo de potência real

\begin{tabular}{c|c|c|c|}
\hline$V_{g}\left[\mathrm{~cm}^{3} / \mathrm{rot}\right]$ & $Q_{\text {teórica }}[\mathrm{L} / \mathrm{min}]$ & $q_{v s}[\mathrm{~L} / \mathrm{min}]$ & Nteórica $[\mathrm{kW}]$ \\
\hline 9,8 & 14,7 & 2,7 & 5,88 \\
\hline
\end{tabular}

Fonte: dos autores (2020).

Com os dados obtidos para a unidade potência, com e sem o uso do acumulador, foi possível realizar uma comparação com relação ao investimento do acumulador. A Tabela 5 apresenta os valores encontrados para os componentes que representam maior valor no sistema e os quais foram o objetivo principal do estudo, bem como o modelo SB330(H) 20A1/112U - 330 A 160 adotado do fabricante de acumuladores HYDAC, com um custo estimado em $\mathrm{R} \$ 10.900,00$.

Tabela 5 - Comparativo do consumo de energia com o uso e sem o acumulador

\begin{tabular}{c|c|c|}
\hline Componente & Custo $[\mathrm{R} \$]$ & Pot. de acionamento $[\mathrm{kW}]$ \\
\hline T7AS B17 & $5.632,20$ & 10,2 \\
\hline T7AS B10 & $4.800,60$ & 6,7 \\
\hline
\end{tabular}

Fonte: dos autores (2020).

Por meio da Tabela 5, é possível identificar o ganho de energia com a utilização do acumulador de pressão, mesmo com investimento inicial deste. Com o uso do acumulador, os custos das bombas seriam também reduzidos, como é apresentado as diferenças entre o modelo B17 para B10. As diferenças da potência consumida é mais de 1.5 vezes maior, sem o uso do acumulador. Essa diferença impacta diretamente na viabilidade econômica do projeto, pois atualmente uns dos maiores valores dos investimentos de um equipamento diz respeito a energia utilizada por este durante sua fase de operação.

\section{VALIDAÇÃO DOS RESULTADOS TECNOLÓGICOS}

Buscando validar a etapa de cálculos analíticos e seleção de componentes, utilizou-se da simulação via software FluidSIM H demo version. Para tal, se faz necessária a definição de algumas condições de operação de cada componente, sendo: rotação do motor: $1500 \mathrm{rpm}$; deslocamento da bomba: $9,8 \mathrm{~cm}^{3} /$ rot.; volume do acumulador: 17,5 litros; pré-carga do acumulador: 160 bar; pressão nominal na válvula de alívio: 240 bar. Na Figura 3 são apresentados os dados entrada para as simulações. 
Primeiramente adicionou-se a bomba hidráulica ao simulador, utilizando os valores obtidos analiticamente, sendo o seu deslocamento de acordo com as condições de operação observadas anteriormente (Figura 3 a). Em seguida, adicionou-se o acumulador, com volume condizente aos cálculos analíticos e uma pré-carga de 160 bar, (Figura 3 b).

Figura 3 - Dados de entrada do software: a) bomba hidráulica e b) acumulador de pressão
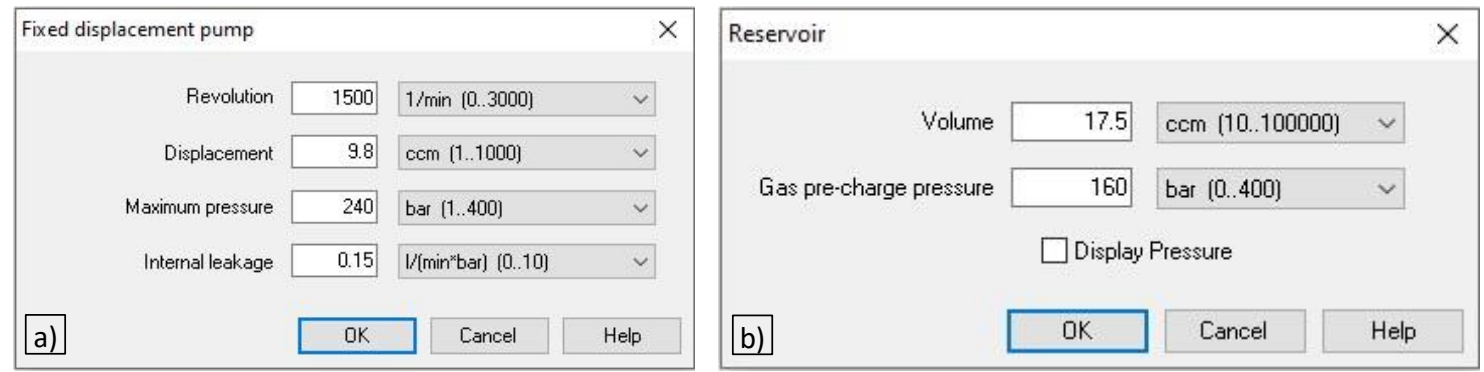

Fonte: dos autores (2020).

Com os dados adicionados, foi possível gerar os seguintes resultados dinâmicos do cilindro hidráulico e da bomba hidráulica em operação (Figura 4).

Figura 4 - Resultados das simulações: a) movimento de avanço e retorno do atuador linear e b) vazão da bomba em instantes de funcionamento
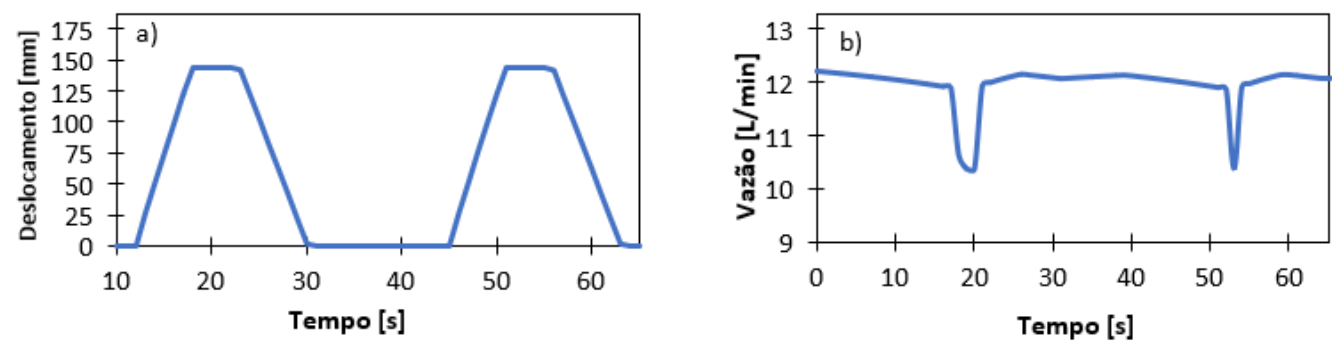

Fonte: dos autores (2020).

Os resultados dinâmicos da Figura 4 facilitam a análise visual do funcionamento do circuito, demonstrando a diminuição de vazão em períodos onde não há ocorrência do deslocamento do cilindro. Isso valida a eficácia da aplicação do acumulador e dessa forma, é possível salientar que a simulação pode ser considerada adequada e satisfatória, condizendo com o funcionamento do circuito hidráulico. Na Figura 4 b, nota-se que durante a parada do atuador, por exemplo, nos tempos de 18 a 23 s, a vazão é aproximadamente a média calculada. Contudo, nota-se que durante o movimento do atuador, há a necessidade da vazão disponibilizada pelo atuador, que somada a vazão da bomba, chega-se à vazão de aproximadamente $12 \mathrm{lpm}$, conforme determinada pela diferença de 14.7 - 2,7 do vazamento.

\section{VALIDAÇÃO DOS RESULTADOS DE ENSINO}

O intuito do trabalho foi avaliar não apenas a parte tecnológica da área específica do curso, mas se a metodologia de ensino aplicada, teve boa aceitação perante aos acadêmicos. Igualmente, se a atividade inicialmente proposta, teve o êxito na compreensão do conteúdo específico da disciplina de sistemas hidráulicos e pneumáticos, além do impacto na aprendizagem dos acadêmicos para sua vida profissional. 
Dessa forma, para validar quali-quantitativamente o potencial da metodologia SATC 2030, bem como dar respaldo sobre a aprendizagem dos acadêmicos, um questionário fora elaborado, o qual envolveu questões sobre a relevância da Atividade Baseada em Problema ABP. O questionário e seus respectivos resultados foram desenvolvidos através de uma plataforma online, e tiveram como participantes, alunos da oitava fase do curso de engenharia mecânica, que normalmente dependem desse conhecimento específico.

As perguntas foram pontuadas em quatro níveis, sendo estes: discordo, indiferente, concordo parcialmente e concordo totalmente. A seguir, as perguntas feitas são apresentadas para a elaboração do questionário, sobre o uso da metodologia para o ensino de engenharia. Nas Figuras 5 são apresentados os resultados obtidos a partir de um total de 15 alunos.

As Figuras 5a e 5b apresentam as respostas das seguintes perguntas: Pergunta 1: A atividade baseada em problemas trabalhou conceitos de soft skills (trabalho em grupo, uso de softwares, estudo autônomo, etc.). Pergunta 2: A atividade baseada em problemas - ABP contribuiu para minha aprendizagem como um todo nos conteúdos da disciplina.

Figura 5 - Resultados da avaliação, como proposta da aprendizagem SATC 2030
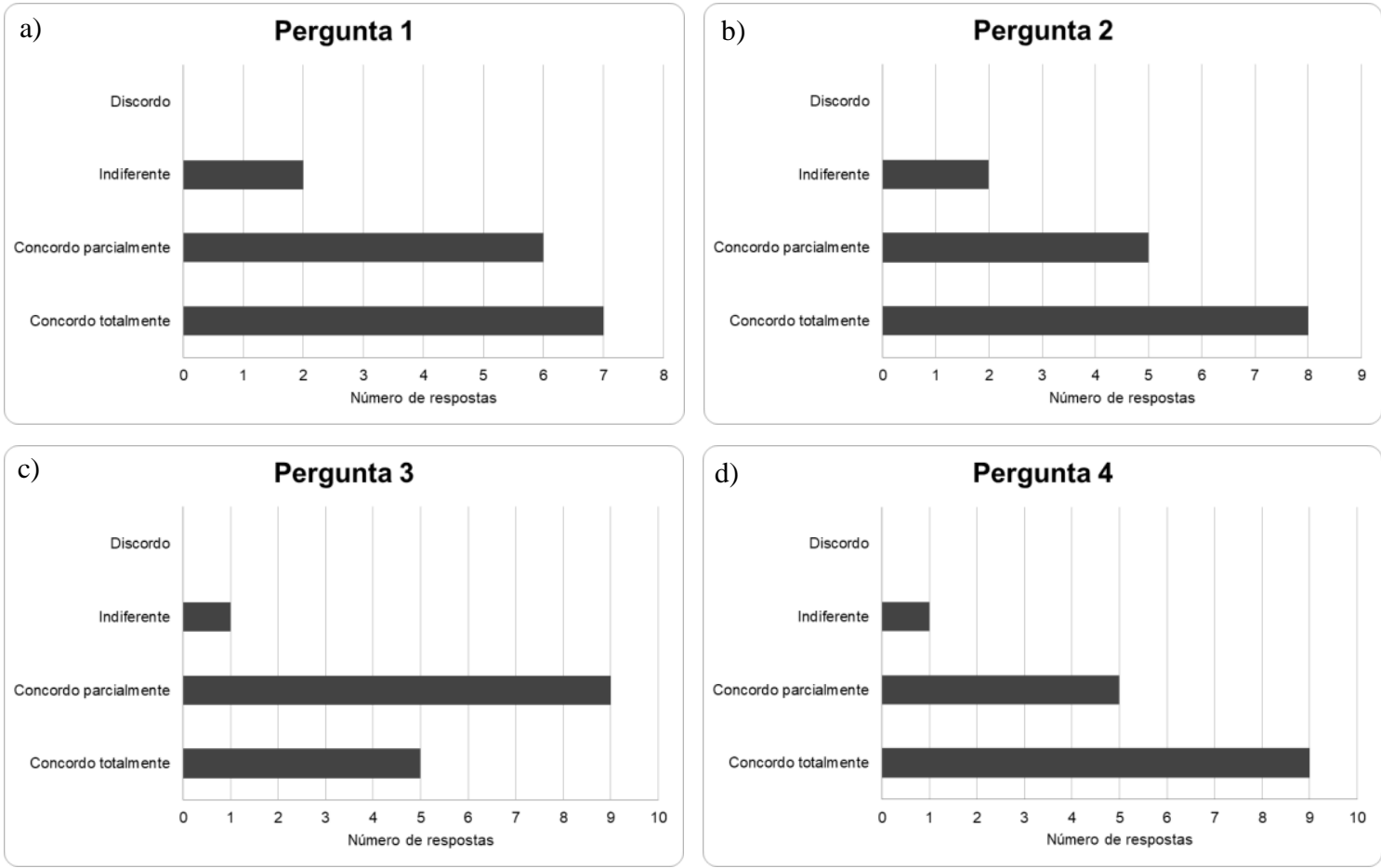

Fonte: Dos autores.

Considerando as perguntas 1 e 2, dos 15 alunos que participaram da pesquisa, mais de $86 \%$ acreditam que a metodologia é relevante e que foi desenvolvido habilidades profissionais e sociais, por meio do uso de software (ex: Figura 3), favorecimento de discussões em grupo, entre outros aspectos de autonomia, como a pesquisa científica que foi usada para a aplicação das equações do próprio conteúdo específico. Apenas, 14\%, mostram que indiferentemente da aplicação da metodologia, o conteúdo foi desenvolvido. Furtado et al, (2018), apresentam resultados de percepção geral dos estudantes da ABP, com relação ao pensamento crítico, interdisciplinaridade e a colaboração do time. Os resultados dos autores mostram que mais de $90 \%$ dos alunos acreditam que esse tipo de atividade seja de maior relevância do que os métodos tradicionais de ensino. 
As Figuras 5c e 5d apresenta as respostas das seguintes perguntas: Pergunta 3: A ABP em questão, despertou um aumento de interesse no desenvolvimento e solução dos problemas propostos. Pergunta 4: A realização da atividade baseada em problemas foi de grande valia no panorama geral.

Para as perguntas 3 e 4, que trataram sobre o interesse na solução do problema e a validação do método, mais de $93 \%$ acreditam que o método seja relevante, concordando parcialmente e totalmente. Especificamente na pergunta 3,5 apenas responderam que concordam totalmente. Isso mostra que ainda existe ainda uma insegurança na resolução de problemas, ou seja, sem auxílio total dos professores. Alguns destes, necessitam que os conteúdos sejam primeiro desenvolvidos de forma tradicional, e a resolução de problemas. Klein e Ahlert (2019), também apresentaram resultados positivos com relação ao interesse, motivação e envolvimento do aluno na disciplina. Os resultados nesse estudos os autores constataram que mais de $83 \%$ dos alunos concordam com atividades baseadas em problemas.

\section{CONSIDERAÇÕES FINAIS}

Primeiramente, o que tange as questões de aprendizado, a problema apresentado ao conhecimento específico, intensificou alguns conceitos da disciplina. Além disso, garante ao discente o exercício de soft skills, características necessárias ao profissional atual e, impacta na eficácia da metodologia de ensino, como sugerem Souza e Dourado (2015).

Analisando os resultados de engenharia a partir das equações e seleção dos componentes apresentados no trabalho, fica evidente que o uso de acumuladores de pressão em sistemas hidráulicos, acarreta a diminuição da potência de acionamento deste, e consequentemente na redução do custo operacional de todo sistema. Os resultados tecnológicos provam também que a metodologia SATC 2030, melhora o conhecimento específico, que é justificado pela coerência entre os cálculos analíticos e da simulação.

Para perspectiva sobre o trabalho, acredita-se que a metodologia poderia ser ainda mais eficiente, caso fosse executada e validade com testes em bancadas, fechando o ciclo de ensino e aprendizagem, ainda revelando respostas mais confiáveis.

\section{REFERÊNCIAS}

CASTELlANOS, Luis Sebastian Mendoza. Modelagem matemática da operação integrada do sistema híbrido Solar/Biomassa, utilizando tecnologias Disco/Stirling, CCP-ORC solar e gaseificação/MCI com sistema de acumulação hidráulico tipo bexiga. Tese (Doutorado) - Faculdade de Educação, Universidade Federal de Itajubá, Itajubá, 2017. FIALHO, Arivelto Bustamante. Automação Hidráulica: Projetos, Dimensionamentos e Análise de Circuitos. $5^{\circ}$ edição, São Paulo: Editora Érica Ltda, 2007.

FURTADO, Anderson Elias; NASCIMENTO, Denise Ferreira Laurito; DE JESUS SILVA, José Wilson. Aprendizagem Baseada em Problemas (PBL) aplicada simultaneamente para estudantes de engenharia de $3^{\circ}$ e $7^{\circ}$ períodos como ferramenta motivacional. Revista Práxis, v. 10, n. 19, 2018.

HYDAC, Catálogo. Acumuladores Hidráulicos de Bexiga. p. 6 e 7.

KLEIN, Niumar André; AHLERT, Edson Moacir. Aprendizagem baseada em problemas como metodologia ativa na educação profissional. Revista Destaques Acadêmicos, v. 11, n. 4, 2020. 
"Os desafios para formar hoje o engenheiro do amanhã"

LINSINGEN, Irlan. Von. Fundamentos de Sistemas Hidráulicos. Florianópolis: Editora da UFSC, 2016. 400 p.

MOREIRA, Ilo da Silva. Sistemas Hidráulicos Industriais. $2^{a}$ edição, São Paulo: SENAISP editora, 2012.

PARKER, Catálogo. Industrial Hydraulic Pumps T7/T67/T6: Denison Vane Technology, fixed displacement. HY29-0001/UK, 2012, p. 10.

PARKER, Catálogo. Hydraulic Pump: T7AS/T7ASW Series Vane Pumps. Catalogue HY29-0008/UK, 2005, p. 4.

PERSON, V., \& BIGNELL, S. Using Problem Based Learning within 3D Virtual World. An Internacional Journal of Industrial Robots, 2011.30 (1), p. 241-246.

REXNORD. Manual de Hidráulica Básica. $3^{\text {a }}$. ed. Porto Alegre: Racine Hidráulica, 1981

SADLO, Gaynor. Using Problem Based Learning During Student Placement to Embed

Theory in Practice. In: The Higher Education Academy, 2014. 2 (1), p. 6-19.

SOUZA, S. C.; DOURADO, L. Aprendizagem Baseada em Problemas (ABP): Um Método de Aprendizagem Inovador para o Ensino Educativo. In: HOLOS, Ano 31, 2015, Rio Grande do Norte. 2015.

TITISARI, K. H.; WIJAYANTI, A.; \& CHOMSATUN, Y. Model Pembelajaran Akuntansi Untuk Meningkatkan Kompetensi Mahasiswa. Jurnal Buletin Studi Ekonomi, (2013). 18 (2), p. 85-190.

\section{PROBLEM-BASED LEARNING APPLIED IN THE SUBJECT OF FLUID POWER SYSTEMS: 2030'S EDUCATION}

Abstract: The learning methodology of Faculdade SATC proposes to teachers that this practice should be conceived in a collaborative and co-creation environment, with the aim of best practices for innovation in higher education. This proposal is related to the new global conjuncture of educational technologies, the breaking of paradigms of traditional education and the new competences and skills required by the labor market. For this purpose, each semester, groups of academics in their respective subject are encouraged to solve real problems, constantly assisted by the professor, who shares their professional experiences in solving these problems. In this work, academics had to evaluate the use of a pressure accumulator in a hydraulic system that operates under intermittent flow and high energy consumption conditions. The activity teaching learning was developed in the subject of fluid power systems, taught in the eighth semester of the mechanical engineering course. At the end, academics answered a questionnaire, to qualitatively and qualitatively validate the application of the methodology, assessing the impact on learning. In the general aspect, the teaching activity provided the resolution of a real problem, applying the knowledge of the specific area, in improving the energy efficiency of the system. Also, the problem-based learning methodology shows itself as a positive evolution to traditional methods, and that promotes the development of students' autonomy.

Keywords: Learning methodology. Innovation in teaching. Fluid power systems. Accumulator. Energy efficiency. 